\title{
LA COLONIA RESISTENCIA COMO MODELO DE FUNDACIÓN REPUBLICANA \\ PROCEDIMIENTOS FUNDACIONALES Y CARACTERÍSTICAS URBANAS
}

\section{Luciana Sudar KLappenbach}

Arquitecta Luciana Sudar Klappenbach. Becaria de Formación de Posgrado Interna de Conicet. Miembro investigador del CEHAU NEA. UNNE. Profesora adjunta interina de la Cátedra de Archivoeconomía en la Facultad de Humanidades. UNNE. Maestrando de Gestión del Patrimonio Arquitectónico y Urbano de la Facultad de Arquitectura, Urbanismo y Diseño de la Universidad Nacional de Mar del Plata.

Cuaderno Urbano N 4, pp. 117-143, Resistencia, Argentina, Diciembre 2004 


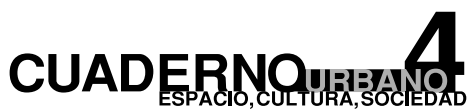

\section{La Colonia Resistencia Como Modelo de Fundación Republicana Procedimientos Fundacionales y Características Urbanas}

\section{Resumen:}

El siguiente artículo analiza la historia urbana de Resistencia tomando aquellos aspectos que incidieron tanto en su fundación como en las características que adoptó su trazado en el mismo período.

El objetivo principal es verificar el proceso de fundación republicana a nivel nacional tomando el caso particular de Resistencia como colonia productiva, e indagar en los aspectos que caracterizaron al urbanismo del siglo XIX.

El análisis se centra en la investigación histórica e integra aspectos devenidos de la situación política-económica, los instrumentos legales, la prácticas disciplinares y los patrones urbanos de fundación liberal.

\footnotetext{
Abstract:

The article deals with the urban history of Resistencia considering those issues dealing with its foundation as with the characteristics adopted by its original layout during the foundational period.

The primary objective is to verify the process of republican foundation at the national level considering the particular case of Resistencia as a productive colony, digging into the aspects that characterized the $19^{\text {th }}$ Century urbanism.

The analysis centers in the historical research pointing to integrate the political economy situation, legal documents, the disciplinary practices and urban standards of liberal foundation.
} 


\section{Luciana Sudar Klappenbach}

La Colonia Resistencia Como Modelo de Fundación Republicana

Procedimientos Fundacionales y CaRacterísticas Urbanas

El abordaje de la historia urbana de la ciudad de Resistencia, centrado en el interés de la lógica que guió su fundación y en la disposición de un determinado tipo genérico que definió su trazado, ha permitido plantar algunas hipótesis que se desarrollarán en el artículo:

* Que la fundación de Resistencia fue producto de la estrategia geopolítica de la incipiente formación del Estado-Nación Argentino, condicionado por los intereses económicos del mercado internacional.

* Que por la utilización de instrumentos jurídico-legales y la aplicación de modelos fundacionales prototípicos en toda la extensión del territorio nacional, devenidos de la aplicación uniforme de resoluciones disciplinares de un cuerpo técnico oficial, se obviaron en muchos casos las realidades locales produciendo una inadecuada ocupación territorial.

* Que las prácticas de mensuras respondieron en mayor parte a los puntos consignados por la legislación que reglamentaba el arrumbamiento de nuevos poblados, habiendo una relación de correspondencia lineal entre el cuerpo legal y el trazado.

* Que en el planteo urbano se traducen elementos propios de la urbanística decimonónica europea.

A partir de estos supuestos y con el objeto de indagar en los factores que determinaron la lógica fundacional de Resistencia y sus características urbanas, a partir de una manifiesta voluntad estatal de fundar la ciudad como medio para llevar adelante el proyecto modernizador de la Argentina republicana, se desarrollarán las siguientes áreas temáticas: luego de la introducción en la que se presenta el marco general de estudio, y el advenimiento del urbanismo que definió una segunda etapa fundacional en la Argentina, interesa exponer en una primera parte, el contexto general político, económico y social de la Argentina Liberal decimonónica, incluyendo particularmente el modelo de estructuración territorial y el marco legal que le dio sustento. En la segunda parte, se introduce a la problemática local del caso de estudio indagando en los aspectos que intervinieron en la fundación del asentamiento como colonia agrícola; el análisis se detiene en las prácticas y técnicas llevadas 


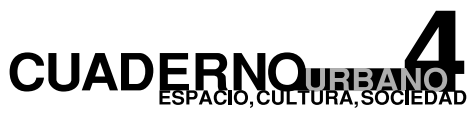

120

\section{La Colonia Resistencia Como Modelo de Fundación Republicana Procedimientos Fundacionales y Características Urbanas}

sobre este territorio con la particularidades que presentaba, y su correlación con el aparato legal que definía las nuevas fundaciones, asimismo queda expuesto el modelo de macrocuadrícula como la manifestación material y el medio pragmático de ocupación territorial, funcional al proyecto modernizador capitalista. Por último, verificamos sobre la estructura racionalizadora del trazado, la presencia de elementos propios de la urbanística del siglo XIX como representaciones del nuevo orden republicano, en un orden simbólico, el cual se expresa en la configuración que fue adoptando el espacio público.

\section{INTRODUCCIÓN}

Abordar un trabajo referido a la historia urbana de Resistencia demanda en principio la necesidad de construir marcos de observación que permitan leer desde diferentes perspectivas tanto el proceso de génesis como de desarrollo del asentamiento urbano. Dada esta premisa se construye el conocimiento intentando analizar a la ciudad desde su concepción como "producto social", donde intervienen todos los procesos de producción humana.

La definición de ciudad como producto social permite acercar la realidad histórica de Resistencia a una relación de correspondencia, y no de consecuencia, con procesos y hechos sociales tan diversos como posibilidades de actuación tiene el hombre en la construcción de su realidad y su historia. Se pretende historiar la ciudad, tomándola como unidad de análisis "con entidad autónoma en sí misma" (Álvarez Mora, 1992: 83), superando de esta manera el concepto de ciudad como mero soporte físico donde suceden hechos sociales. Se entiende a la ciudad como el lugar en que, parafraseando a Luis Alberto Romero, "...se cruzany adquieren una forma específica todos los procesos de la sociedad, y allí se requieren todos los enfoques y marcos analíticos disponibles para los historiadores." (Romero L. A. En Cacopardo, F. 2002: 11).

El urbanismo del siglo XIX en América Latina ha presentado diversas características correspondientes a los momentos históricos y a los modelos políticos económicos dominantes. Bajo esta mirada la ciudad de Resistencia emerge dentro de un proceso en el que se articu- 


\section{Luciana Sudar Klappenbach}

La Colonia Resistencia Como Modelo de Fundación Republicana

Procedimientos Fundacionales y CaRacterísticas Urbanas

lan aspectos políticos, económicos, sociales y disciplinares, presentándose como un modelo paradigmático de ocupación territorial en la etapa republicana argentina.

Hasta la primera mitad del siglo XIX, continuaba vigente el modelo hispánico de ocupación territorial caracterizado por la inercia y la carencia de proyectos de estructuración territorial, que respondía más a la herencia de desarticulación propia del modelo mercantilista impuesto por el imperio español en América que a las estrategias de planificación territorial impulsadas por los nuevos Estados-Nación europeos emergentes de la revolución industrial. La disposición territorial en Argentina se caracterizó durante esta época por la incomunicación y el aislamiento de las ciudades acorde con los esquemas que planteaba una economía regional de subsistencia. Durante este período no se fundaron nuevos pueblos, la vinculación territorial mantenía los antiguos caminos de la colonia, especialmente orientados hacia Perú, con predominio de las zonas de Cuyo, Centro y Noroeste del país. La región del Litoral por su parte, con centro en Puerto de Buenos Aires y con vinculación fluvial sobre el Este del territorio comenzó a pesar recién a partir de la creación del Virreinato del Río de la Plata (1776), poco antes de la independencia.

Recién a partir de la segunda mitad del siglo XIX se planteó un cambio sustancial en la urbanística argentina como parte de un proyecto mayor de estructuración territorial, en respuesta al modelo agroexportador por el cual el incipiente Estado-Nación argentino se integró al capitalismo mundial. Dentro de las transformaciones territoriales y urbanas se presentaron casos disímiles, por una lado aquellas que se produjeron sobre las estructuras urbanas coloniales existentes en los "ensanches" (Tucumán, Buenos Aires, Córdoba), y por el otro, las de nueva fundación republicana, en la que se identifica una serie de variantes acordes al rol que les tocaba asumir a estos asentamientos en el nuevo sistema político, económico, social y administrativo de la Argentina "unificada".

Estas variantes estaban determinadas por los objetivos y funciones que asumía cada nuevo asentamiento tal como administrativos y de servicios (capitales de provincia) como el

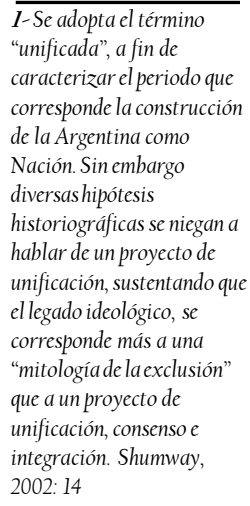




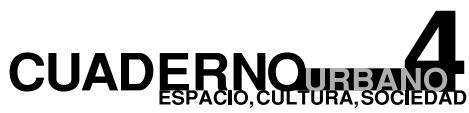

122

\section{La Colonia Resistencia Como Modelo de Fundación Republicana Procedimientos Fundacionales y Características Urbanas}

caso de La Plata en nuestro país; recreacionales, como Mar del Plata, Necochea; militares que acompañaron el proceso de extensión de fronteras al Norte y en la Patagonia; y aquellos con roles marcados por la producción, los cuales poseían distintas características propias al sistema productivo a los que pertenecían, los que definían "cierto tipo genérico repetido" (Nicolini, 2000: 8): agrícola-ganadero (Pcia. de Buenos Aires, Chaco, la Pampa), explotación forestal (Chaco y Norte de Santa Fe), industriales (Ingenios de Tucumán); nodos comunicacionales: ciudades del ferrocarril (Colonia Caroya, Sampacho) o portuarias (Barranqueras, Colón, Ingeniero White).

La ciudad de Resistencia, como tantas otras ciudades argentinas, no fue más que el producto de la integración de estos factores ideológicos, políticos, geopolíticos y económicos puestos de manifiesto en la república. Surgió en un contexto determinado por el modelo de organización nacional, en el que debía compatibilizarse el nuevo sistema económico: el capitalismo, la división internacional del trabajo y el rol que pasó a desempeñar la Argentina, dentro de un marco político de democracia federal. A ellos se pueden sumar otros componentes que constituyeron la base de esta organización, especialmente en la estructuración y expansión de los territorios: el poblamiento de áreas desocupadas mediante un sistema racional de urbanización y distribución de áreas productivas; el fomento de la inmigración y el tendido de ferrocarriles. Finalmente, debemos agregar que además de representar y dar respuesta a una temática predominantemente productiva, se hicieron presentes también elementos que definieron al urbanismo del siglo XIX, tanto en el planteo morfológico urbano (traza, paisaje), como en los factores funcionales: nuevas actividades y localización, nuevos usos sociales, infraestructuras, equipamiento urbano, y factores ambientales estéticos.

\section{LA ARGENTINA LIBERAL}

En el plano internacional hacia 1870, Inglaterra, Francia, Alemania y EEUU se aprontaban a entrar en la Segunda Revolución Industrial, en tanto en el Río de la Plata se vivía una at- 


\section{Luciana Sudar Klappenbach}

La Colonia Resistencia Como Modelo de Fundación Republicana

Procedimientos Fundacionales y CaRacterísticas Urbanas

mósfera de sociedad periférica colonial y tecnológicamente primitiva. La generación de la Organización Nacional encontró no sólo un país desértico y dividido, sino también introvertido y tecnológicamente atrasado.

Al hablar de la Argentina liberal, se hace inmediata referencia a la generación del ` 80 , si bien muchos historiadores hablan del modelo del ' 80 , habría que replantearse si ésta existió como tal, ya que reconocemos los antecedentes ideológicos que movilizaron esta etapa en la Argentina en la generación del 37, con los primeros pensadores de una política liberal: Sarmiento, Alberdi, Echeverría, entre otros. ${ }^{2}$

Lo que caracterizó a la generación política de las últimas décadas del 1800 sin dudas, fue la suficiente claridad conceptual que poseían, en el plano doctrinario, para sostener una sucesión ininterrumpida de administraciones de gobierno, orientadas por los mismos objetivos e instrumentos, los cuales se pueden puntualizar a modo de introducción a la problemática territorial:

1. Pacificar al país: promoviendo su unidad, a partir de la superación de la antinomia unitarios-federales y el surgimiento de nuevas estructuras partidarias.

2. Integrarse al resto del mundo: aspiraba a integrar al país a la región más evolucionada del planeta: Europa, particularmente Gran Bretaña. Esto implicaba: aceptar la división internacional del trabajo y elaborar una estrategia de desarrollo hacia afuera.

3. Modernizar la estructura productiva del país, especialmente en el sector más relevante: el agropecuario.

4. Aumentar la disponibilidad de factores de producción: la tierra (conquista al desierto), el capital y trabajo.

5. Crear el marco jurídico para el tránsito de la sociedad colonial al país moderno (creación de códigos: civil, comercial, etc.).

6. Desarrollo de una infraestructura básica: ferrocarriles y puertos.
2. "Ninguno de ellos creyó que el combate sería ganado en su generación, antes bien se ocuparon de crear un marco ideológico, de fundar mitos, en palabras de Halperin Dong hi, que a los futuros gobiernos les permitieran avanzar hacia la prosperidad y la democracia bajo un régimen constitucional." Shumway, 2002: 165 . 


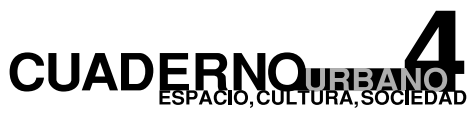

124

La Colonia Resistencia Como Modelo de Fundación Republicana

Procedimientos Fundacionales y Características Urbanas

La división internacional del trabajo: su implicancia en la construcción de un modelo de organización nacional:

El nuevo sistema económico, el capitalismo y el librecambio, devenido de la Revolución Industrial, la mayor disponibilidad de capitales y la creciente capacidad de las metrópolis para absorber las exportaciones latinoamericanas, se tradujeron en una mayor vinculación de Latinoamérica dentro del circuito de la economía mundial.

La división internacional del trabajo establecía para Hispanoamérica y áreas subdesarrolladas (colonias y factorías en Asia y África durante los siglos XVIII y XIX) el rol de productores de materias primas y la posibilidad de un nuevo mercado de consumo. Gran Bretaña, que se había convertido en la potencia dominante, era fundamentalmente quien manejaba este juego de roles, disponiendo a su vez del capital, la tecnología y del manejo del sistema de transporte marítimo, lo que implicaba la movilización del mercado, productos y capitales.

Tulio Halperin Donghi, en su libro "Historia contemporánea de América Latina”, sostiene esta tesis, definiendo a este período como neocolonial "...el proceso que llena la etapa iberoamericana comenzada a mediados del S. XIX: la fijación de un nuevo pacto colonial...este nuevo pacto transforma a Latinoamérica en productor de materias primas para los centros de la nueva economía industrial, a las vez que de artículos de consumo alimenticio en las áreas metropolitanas, la hace consumidoras de la producción industrial de esas áreas..." (Halperin Donghi, 1996: 223)

En el período comprendido entre la unificación nacional y la primera guerra mundial (1862 - 1914) el país experimentó un sorprendente crecimiento económico asociado a varios factores: desarrollo de actividades productivas como la ganadería y la agricultura, privilegiada condición de las tierras en la pampa húmeda, incorporación de capitales y fuerza de trabajo extranjeros. Estos dos últimos manifestados en la construcción de ferrocarriles y el fomento de una política migratoria. 


\section{Luciana Sudar Klappenbach}

La Colonia Resistencia Como Modelo de Fundación Republicana

Procedimientos Fundacionales y CaRacterísticas Urbanas

En síntesis, en Argentina tres factores definieron su dinámica de crecimiento y desarrollo:

1. Luego de la conquista al desierto (Patagonia y Bs. As.) y del Chaco, se disponía una inmensa superficie de tierras que quedaban marginadas y desconectadas de los centros productivos y de desarrollo (Litoral y Buenos Aires) y así también del puerto. El ferrocarril fue entonces el elemento estructurador del territorio y consecuentemente de las economías regionales. Con este novedoso sistema de transporte se acortaban las distancias y se reducían los costos de traslado.

2. El segundo factor importante fue el del capital — foráneo, en la mayoría de los casos- se realizaron grandes inversiones orientadas fundamentalmente a la construcción de infraestructura: ferrocarriles y puertos, aunque también se utilizaron para mejoras en la explotación, también se incorporaron maquinarias y tecnología.

3. El tercer factor fue la mano de obra; así como se importó capital y tecnología, llegó a nuestro país una gran ola migratoria proveniente de Europa movilizada por la difícil situación y casi nulas posibilidades de crecimiento que sufría este continente, como consecuencias de la revolución industrial. Este gran número de inmigrantes permitía a nuestro país el poblamiento efectivo de las nuevas tierras y la incorporación de fuerza de trabajo para el desarrollo de la creciente actividad agropecuaria y de las nuevas funciones que se incorporaban.

\section{El proyecto de estructuración territorial: Colonización Republicana}

La unificación del interior e integración de las economías regionales, sumadas a la extensión de vías férreas y al incentivo de una economía productora primaria con el empleo de la mano de obra inmigrante, acarrearon la aparición de nuevos poblados y la conformación de colonias que respondían a distintas necesidades o actividades. 


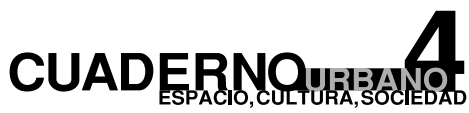

126

La Colonia Resistencia Como Modelo de Fundación Republicana
Procedimientos Fundacionales y Características Urbanas

Ley Nacional de Inmigración y Colonización:

La ley 817 (1876) sancionada durante la presidencia de Avellaneda instrumentó los diferentes mecanismos para la implementación de una política de fomento a la inmigración europea:

* Instalación de oficinas especiales o consulados.

* Otorgamiento gratuito de pasajes hasta destino final

* Alojamiento a cargo del Estado durante los primeros días de su llegada.

En esta ley quedaban claramente expresas las intenciones colonizadoras de la generación del ' 80 , y la implementación de una política inmigratoria para el logro de esos fines. No sólo fijaba las condiciones para la adjudicación de tierras, sino que establecía cómo debía llevarse la administración de las colonias y las características del trazado. Se creaban también el Dpto. General de Inmigración y la Oficina de Tierras y Colonias a cargo de quien estaba asignada la exploración de los territorios nacionales, la elección de los terrenos, mensura, subdivisión de lotes y trazado de las colonias.

Por la primera se establecía: “...proteger la inmigración honorable y laboriosa, fomentar su internación en el país, proveer por cuenta de la Nación el embarque y transporte de los inmigrantes que quisieran internarse dirigiéndoles a los puntos que el Poder Ejecutivo designara para colonizar". "Ser alojados y mantenidos hasta tanto fuesen enviados a destino y trasladados al punto de la República donde quisieran radicarse, introducir libres de derechos las prendas de uso, vestidos ... herramientas y útiles del arte y oficio que ejerciera". (Miranda, 1968: 7)

Dentro del período de colonización del siglo XIX hubo una diferenciación en la estructuración de las colonias determinada por los objetivos a que debían responder y su funcionalidad respecto a la productividad y organización del territorio nacional. 


\title{
Luciana Sudar Klappenbach
}

La Colonia Resistencia Como Modelo de Fundación Republicana

Procedimientos Fundacionales y CaRacterísticas Urbanas

\section{LA CIUDAD DE RESISTENCIA:}

\section{Fundación}

\begin{abstract}
"Se puede afirmar que esta actual capital, y esta actual provincia surgieron a la vida argentina en momentos especiales para el quehacer nacional. Son fruto de la transformación política, económica, social, cultural y educacional que se operaba para el crecimiento de un país que había permanecido estacionado justamente por la inmadurez de la adolescente vida republicana. Del período de afianzamiento en moldes liberales y europeos..." (Vázquez Gualtieri, 1978: 85)
\end{abstract}

Este período queda identificado como aquel en el que se construyeron los mecanismos legales y técnicos de aplicación a las nuevas fundaciones y se efectuaron las prácticas reales sobre el territorio, en el cual podemos comprender cuáles fueron las lógicas históricas que definieron su fundación. Merece el análisis la correspondencia entre las leyes que actuaron como reguladoras y las prácticas llevadas a cabo por un lado, y por el otro, las características de este trazado dentro del nuevo esquema urbano.

Resistencia es un auténtico paradigma del modelo colonizador impulsado por los hombres de pensamiento liberal de la Argentina del S XIX. Es uno más de los modelos prototípicos que se habían diseñado para la efectivización del poblamiento y explotación de las nuevas tierras ganadas a los aborígenes de la Patagonia y del Gran Chaco.

Los Territorios Nacionales del Chaco fueron la materialización del espíritu de la Constitución de 1853, que velaba por la integración y unificación de los territorios argentinos, y el resultado de la concreta acción de los hombres del '80. “...Aparece y se empina en la vida pública argentina en el momento de la realización del programa constitucional, en que el país abría las puertas a todos los hombres del mundo que quisieran habitar el suelo argentino, gozando de los beneficios de la libertad. Irrumpe en el acontecer social, político y económico conjuntamente y como efecto propio de una 


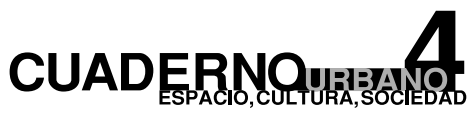

128

\section{La Colonia Resistencia Como Modelo de Fundación Republicana Procedimientos Fundacionales y Características Urbanas}

3- Ley №686 "Ley relativa al Gobierno de una Parte de los Territorios Nacionales del Chaco". Registro Nacional de la República Argentina. Tomo VII. Años 1874 1879, pgs. 111, 112

4-Villa Occidental dependió del Gobierno Argentino hasta 1879, fecha en que, por arbitraje, pasó a serpartedel Territorio Paraguayo. legislación coherente, progresista y trascendental, que nos da la imagen de Nación Integrada." (Rossi, 1997: 69).

La colonización y organización del Chaco tuvo como objetivo, además de los intereses económicos dar respuesta a una necesidad política de apropiación de territorios ante la disputa de esas tierras con el gobierno de Paraguay. Debido a los sucesos de la Guerra de la Triple Alianza (1865-1870), junto al no menos importante aspecto del interés de explotación económica, llevaron a que el presidente Sarmiento pensara en un asentamiento militar en las fronteras y la dominación efectiva, a través del poblamiento de los territorios nacionales. Para efectivizar estas intenciones se pusieron en vigencia varias leyes, en las cuales se manifestaron las ideas liberales de la época con el concepto civilizador de Sarmiento y el afán colonizador de Avellaneda.

En el marco de la ley 576 de 1872, durante la presidencia de Sarmiento se creó la Gobernación del Chaco, y se nombró primer gobernador del Territorio del Chaco al Gral. Vedia, asentando la residencia de la gobernación en Villa Occidental (Hoy, Villa Hayes, Paraguay).

Esta Ley sentó las bases para la organización política del Chaco. Por la misma, en el Art. 10 se ordenaba practicar la mensura de los terrenos adyacentes a cada sección poblada, y se determinaba la disposición de la tierra por un sistema uniforme (Punto 4ํ․ Art. 10 de la ley 576).

Dos años después, por la ley $686^{3}$, se creó la Jefatura Política del Chaco en el territorio comprendido entre el río Bermejo y el Arroyo del Rey (Chaco Austral) y se ordenaba el establecimiento de cantones militares sobre la margen derecha del río Paraná para la traza de nuevos pueblos, construcción de ciudades como medio de pacificación y de colonización del territorio.

La ley marcó también la división del Chaco Boreal y Austral, éste último pasó a depender del Poder Ejecutivo Nacional y ya no de Villa Occidental ${ }^{4}$. En 1875 el presidente Avellaneda 


\section{Luciana Sudar Klappenbach}

129

La Colonia Resistencia Como Modelo de Fundación Republicana

Procedimientos Fundacionales y CaRacterísticas Urbanas

nombró jefe político del Chaco a Aurelio Díaz, y secretario al mayor Jorge Luis Fontana, en cumplimiento de la ley 686.

Prácticamente diez años después, al sancionarse la ley 1532 en 1884, se creó la Gobernación del Chaco, quedando el Territorio del Chaco en dos gobernaciones: la de Formosa y la de Chaco. Resistencia se constituyó de hecho en la Capital de la Gobernación al ser el único municipio, a la vez que colonia y cantón de asentamiento militar.

La fundación de Resistencia se hizo finalmente efectiva con la llegada de 43 familias friulanas que desembarcaron en el río Negro en enero de $1878 .{ }^{5}$

Vista la correspondencia del surgimiento de un nuevo asentamiento urbano como parte de un proyecto nacional, que a su vez respondía directamente a la movilidad económica internacional, queda por presentar cuál fue la relación entre el trazado, la prácticas técnicas, y los mecanismos legales que se implementaron y condicionaron el trazado de Resistencia, así como también cuales fueron las características en el orden del diseño urbano del período de estudio.

Las instrucciones para la fundación de las colonias estaban totalmente regladas por la legislación, el análisis del trazado como artefacto material permite leer una correspondencia en el plano con los trazados teóricos previstos en el departamento de Ingenieros, durante los diez años que demoró la aprobación de la mensura definitiva; sin embargo en cuanto a la realidad espacial, funcional, y paisajística era poco lo que tenía que ver con tan planificada ordenación.

En Resistencia, existieron realidades locales que definieron situaciones que no podían compatibilizarse con las indicaciones oficiales. Cabe destacar, entre éstas, las características topográficas y geográficas, que no respondían a las condiciones requeridas para el emplazamiento de los nuevos asentamientos, también presentaban dificultades tanto para los $\overline{5-\text { La fecha simbólica que representa }}$ este momento fue tomada como la del 2 de febrero de 1878, arraigada en la conciencia ciudadana, sin embargo se conoce por investigaciones realizadas que la fecha real de desembarco fue el 27 de enero de 1878 (Maeder, 1996.) 


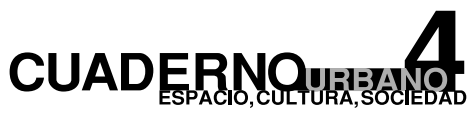

130

\section{La Colonia Resistencia Como Modelo de Fundación Republicana Procedimientos Fundacionales y Características Urbanas}

6-Debe considerarse que el patrón ideológico de la época se fundaba en el paradigma del pensamiento iluminista de la cultura moderna, tal como lo expone Barreto la relación entre el hombre y la naturaleza se daba mediante la dominación y la explotación del primero sobre la segunda, basada en el

racionalismo científico que sostenía que el hombre y su razón poseían el controlyel

conocimiento del universo. Barreto, 1998: "El medio ambiente, el subdesarrolloy la ciudad. Algunas líneas de acción para una política urbana ambientalistay social" En:Propuestaecológica. № 21, Posadas. trabajos de mensura como para su adaptación a las reglamentaciones oficiales. "Se pretendían zonas altas sobre vías de comunicación fácil, preferentemente cerca de poblaciones ya existentes sobre la margen contraria del Río Paraná, que contara con tierras aptas para la agricultura y donde el agua potable fuera de fácil extracción." (Leiva, 1981:24), a esto de sumaban las desarticulaciones que existieron en el reparto de la tierra pública.

El proceso de fundación de las colonias chaqueñas (Resistencia fue la primera fundada en Territorios del Chaco), se realizó en forma sincrónica respecto del período de consolidación del aparato legal en materia urbanística, el cual cambió y evolucionó en un lapso de pocos años. Esta fue otra de las causas que demandaron la necesidad de realizar más de una mensura, a fin de cumplir con lo estipulado por la ley.

El aprovechamiento y la racionalización del suelo rural y urbano encontraron respuestas en la aplicación de un sistema de redes, organizado en una macrocuadrícula territorial, que ofrecía una alternativa en cuanto a la organización de las actividades productivas agropecuarias (encuadre en la realidad agro exportadora argentina) y una distribución equitativa de la tierra (Cacopardo, 2000: 166). En este momento "las ciudades ya no son puntos aislados de avanzada para la conquista al territorio, son precedidas de un sistema racional, geométrico y abstracto que las integra". (Cacopardo, 2000:166). Este planteo suponía una semejanza en todo el territorio de las condiciones productivas, hecho que en el caso de Resistencia verificó su ineficiencia, o tal vez una debilidad al pretender homogeneizar y generalizar situaciones ignorando los contextos particulares. En este caso la elección de los terrenos no fue el propicio para la efectivización de este tipo de modelos, ya que la mayor parte de los terrenos mensurados resultó inapropiada para el desarrollo de la actividad agrícola. ${ }^{6}$

Otro factor que queda expuesto y resulta una invariante del proceso colonizador es la aplicación de modelos prototípicos en toda la extensión del territorio mediante la utilización de instrumentos jurídico-legales, en cuyo concepto se obviaban las realidades locales produciendo una ocupación física territorial sistemática y racional. ${ }^{7}$ 


\section{Luciana Sudar Klappenbach}

La Colonia Resistencia Como Modelo de Fundación Republicana

Procedimientos Fundacionales y CaRacterísticas Urbanas

El análisis de los procedimientos técnicos (mensuras) realizados en la Colonia Resistencia, que se exponen a continuación, permiten presentar las experiencias en estos territorios. Agrego, sin embargo, la identificación de una intención clara en proponer desde una política oficial un modelo de ocupación territorial, tan racional que pudiera ser aplicado a cualquier geografía del país. Si observamos los planos para Formosa, Balcarce, Tandil, y Resistencia, entre otros, se dificulta la posibilidad de hacer una rápida identificación, esto devela la presencia de una estructura dominante ejercida sobre cualquier territorio. Al desarrollar un análisis en otro nivel de focalización, sí podríamos identificar las diferencias que subyacen a cada uno de los casos, por ejemplo diferenciación en el número de manzanas destinadas a plazas, número de plazas, límites, condiciones topográficas, etc.

\section{Emplazamiento y mensura:}

Fueron tres leyes fundamentales que actuaron en las mensuras de la Colonia Resistencia: la primera del año 1872, la segunda, de 1874 y la última del año 1876, también fueron tres las mensuras realizadas: 1875, 1878 y 1884 . Entre el avance legal y técnico de fundación de poblados y las mensuras realizadas existe una correlación sincrónica, no obstante se presentan algunas diferencias devenidas principalmente de las Instrucciones de la Comisión de Obras Públicas de la Nación y de algunos aspectos físicos que condicionaron el cumplimiento estricto de lo estipulado por la ley.

Si nos remitimos a la legislación que reglamentaba las mensuras, y las realizadas efectivamente sobre estos territorios (Colonia Resistencia), encontraremos un paralelismo, fácilmente perceptible en la observación de los planos. La primera mensura (Fig.l) realizada por Seelstrang, Foster y ayudantes refleja los puntos expuestos con lo dispuesto en la ley 686, decreto de 1875, Art. $3^{\circ}$ (Fig. 2). No obstante, se presentaron algunas modificaciones respecto a esta. En esta primera disposición, se concedía una superficie menor para la creación de la colonia, cuatro leguas cuadradas, que lo dispuesto en la ley 817 de 1876; sin embargo debe destacarse que la ubicación del pueblo respecto de la sección no se encontró en el centro de la misma sino en el extremo este de la colonia. Otro punto que no coinci-

\footnotetext{
7-En este sentido quiero dejar expuesta la hipótesis planteada por Cacopardo: "los trazados de los pueblos no obedecieron sólo a una rígida trama racional, que se baja desde arriba a modo de un sello neutro y repetitivo. Si bien se puede hablar de un tipo general, es en las variaciones sutiles, posibles en el estricto marco de lo que se podía pensar en ese momento donde es posible observar nuevas concepciones."

Aparentemente, esto podría significar una contradicción con lo expuesto, no obstante leo en esta postura la necesidad de definir planos de análisis, capas que permitan remitir a observaciones particularizadas dependiendo del acercamiento a cada uno de los casos. CACOPARDO, F. "Ciudady territorio en el siglo XIX: de la macrocuadrícula territorial a la manzana". En Cuadernos de Historia Urbana I. Facultad de Arquitecturay Urbanismo. Universidad Nacional de Tucumán. 2000: 166.
} 


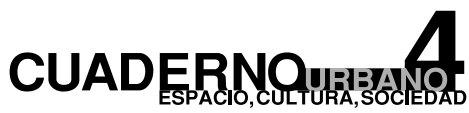

132

\section{La Colonia Resistencia Como Modelo de Fundación Republicana Procedimientos Fundacionales y Características Urbanas}

\section{Primer Mensura 1875}
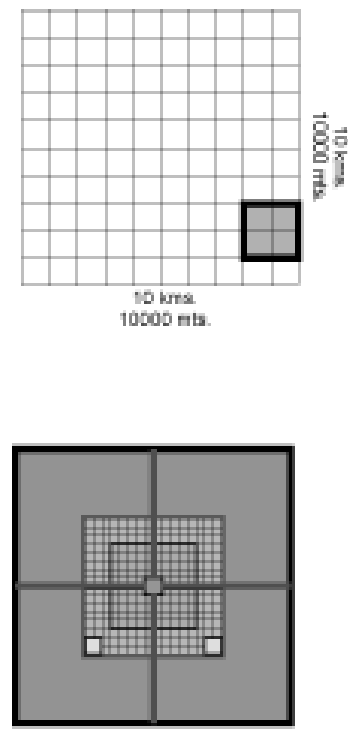

Fig. 1

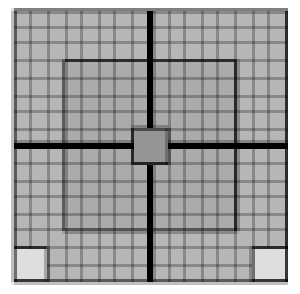

dió con la legislación fue el referido a las dimensiones de las chacras, mientras que ésta determinaba una superficie de 400mts. x 500mts., en la mensura se definieron lotes rurales de 100 has $=1000 \mathrm{mts} . \mathrm{x}$ $1000 \mathrm{mts}$. En esta mensura, aunque no lo indicaba la ley, también se estratificó una escala para las circulaciones, definiendo así una jerarquía.

Estas diferencias las debemos a las instrucciones dadas por la Comisión de Obras Públicas de la Nación en 1875 (Colazo, 1975: 132) que en muchos puntos no coincidían con la Ley; tal es el caso de la dimensión de los lotes para chacra, la jerarquización de vías de circulación, así como la disposición de plazas en los ángulos sur y oeste en el perímetro exterior de las quintas.

En las mensuras siguientes, ya bajo las Instrucciones de la Ley 817 (Fig. 3), realizadas primero por el agrimensor Juan Dillon (Fig. 4) y posteriormente por Carlos Tassier (Fig. 5), se manifestó un incremento de superficie total, en función de los dispuesto por la ley. Es así que la Colonia queda conformada dentro de una sección ${ }^{1}$ de aproximadamente 40.000 has.

El trabajo de Tassier se focalizó en la entrega de las tierras y en la medición y amojonamiento del sector suroeste. Sin embargo introdujo modificaciones en el pueblo, reubicando las plazas que se preveían en los extremos de la sección de pueblo en los vértices definidos por el primer anillo de circunvalación que separaban los lotes de pueblo de los de quintas.

Las diferentes mensuras introdujeron algunas variantes, aunque fue el trabajo de Seelstrang y Foster el que definió el trazado general por lo que la planta urbana quedó definida sobre un total de 256 manzanas, correspondiente a un perímetro de dieciséis manzanas por dieciséis. 


\section{Luciana Sudar Klappenbach}

La Colonia Resistencia Como Modelo de Fundación Republicana

Procedimientos Fundacionales y Características Urbanas

Otro punto a analizar es el referido a la ubicación de la plaza Belgrano. Si observamos hoy un plano de la ciudad vemos que ésta se localiza con un desplazamiento de una manzana hacia el noroeste desde donde se había determinado en las mensuras. Este desplazamiento se debió a una extraña operación inmobiliaria fechada el 25 de noviembre de 1891, en la cual Carlos Boggio — uno de los pioneros en el poblamiento de la colonia - permuta el local que había sido construido como sede municipal, ubicado en la manzana 133, solar D, por la manzana 52 (solar destinado a plaza, según las mensuras). "Al efectuar dicha permuta, Boggio afirmaba que se hallaba frente a la Plaza, terreno que el gobierno nacional había adjudicado a Luisa Charriau de Boggio, su esposa, con fecha ll de marzo de 1889. En realidad, el terreno destinado para plaza, por ese entonces ocupado por una toldería de indios vilelas, era la manzana inmediata... había sido adjudica por error a la esposa de Boggio, por cuanto dicha manzana estaba destinada a plaza pública, según el trazado que había aprobado el Poder Ejecutivo Nacional." (Piacentini, 1970: 266)

Queda en este caso explícito cómo algunas condiciones locales -en este caso un error en las operaciones de asignación de lotes a los colonos- produjeron cambios estructurales en la definición de una trama urbana.

\section{EL DESARROLLO DE LA COLONIA Y LAS CARACTERÍSTICAS DEL URBANISMO LIBERAL:}

\section{Centralidad y lógica ocupacional:}

La concepción espacial de la Colonia dejaba claramente expresa la intención de dotar un área central, que valiera de servicio a las chacras destinadas a la producción agrícola. Estipulaba también una transi-

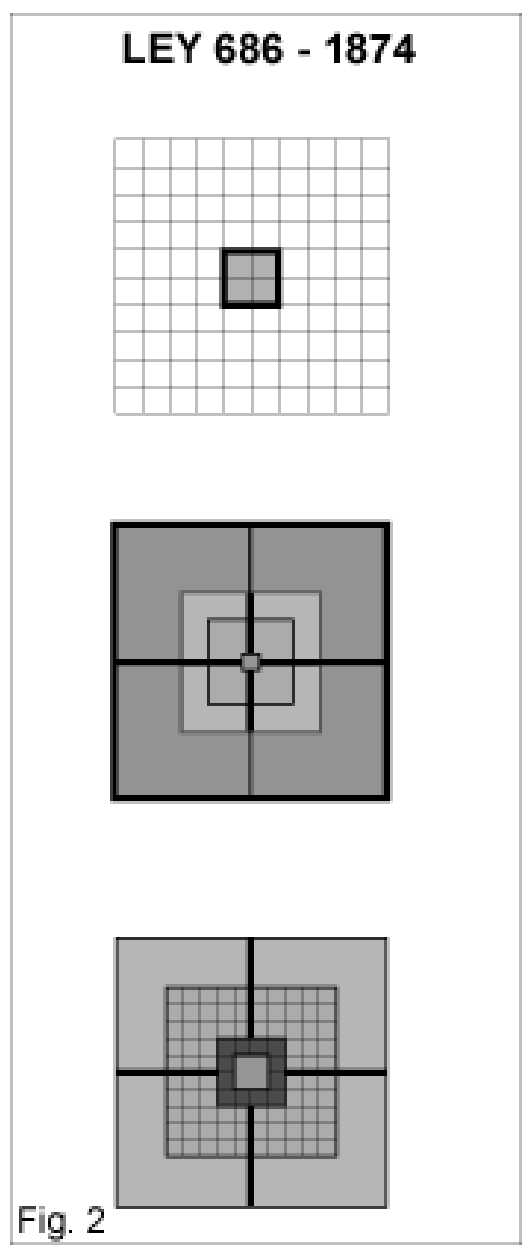




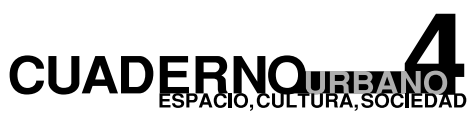

134

\author{
La Colonia Resistencia Como Modelo de Fundación Republicana \\ Procedimientos Fundacionales y Características Urbanas
}

LEY 817 - 1876
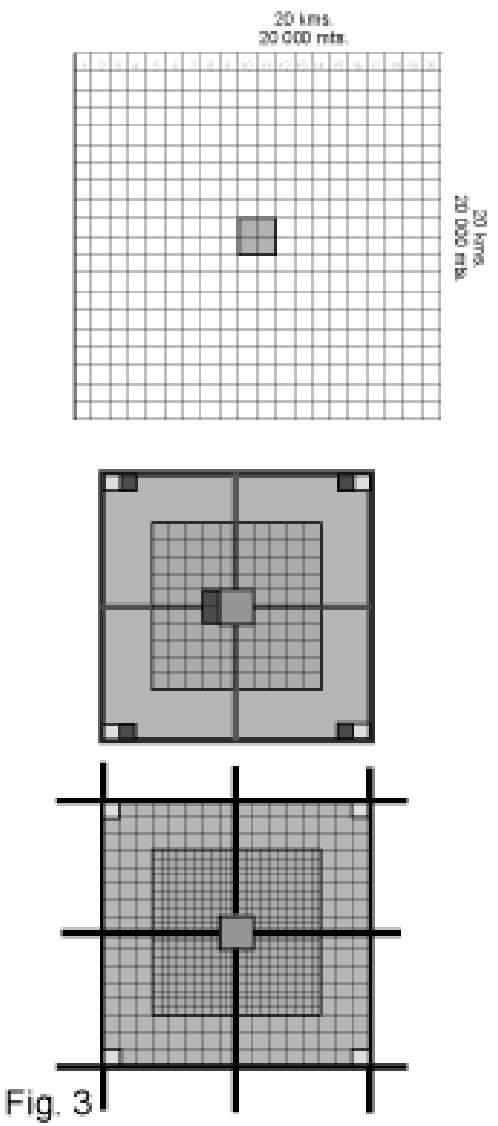

ción gradual entre los lotes centrales, urbanos, y la periferia productora (Fig. 6). Los usos del suelo urbano se determinaban entonces por la mayor o menor densidad de ocupación. Es así que se previó para el centro como unidad urbana la manzana de $100 \mathrm{mts}$. de lado subdividida en lotes de 50 x 50mts. Las quintas se definían por la utilización completa de la manzana, y luego las chacras de 100 has para las actividades extractivas. Esta condición de centralidad y la consolidación de ese centro se vieron sustentadas por un lado por las operaciones físicas de trazado urbano y la perduración de esa estructura urbana original, y por el otro por la aglutinación de factores de orden funcional (en términos de localización de actividades) como los que hicieron a las intervenciones urbanísticas: provisión de infraestructuras, equipamientos, estética urbana, etc.

La colonia, en este estadio, se presentaba como centro urbano total de servicio a la periferia agrícola inmediata, periferia tal que sería ganada en el futuro por los procesos de urbanización y quedaría incorporada a la ciudad actual (cumpliéndose de esta manera la posibilidad que planteaba la macrocuadrícula como sistema potencialmente urbanizable). La solidez en el planteo de centro original, delimitado perfectamente por las avenidas de circunvalación y por la estructura urbana permite que sea reconocido, y de hecho lo es como centro aún en la actualidad.

La particularidad de este modelo radica en la imposición de un planteo regular, sobre un terreno que si bien presentaba una topografía chata, se hallaba rodeado y salpicado de numerosas lagunas, con manchones de monte y con límites muy fuertes hacia el noreste, constituido por el río Negro y hacia el suroeste por el riacho Arazá. En este aspecto, se pensaba que las lagunas podían y debían ser rellenadas, y que todos los inconvenientes que pudiera presentar el lugar se superaría dominando la naturaleza. 


\section{Luciana Sudar Klappenbach}

La Colonia Resistencia Como Modelo de Fundación Republicana

Procedimientos Fundacionales y Características Urbanas

Sobre un terreno accidentado se superpuso esta trama homogénea y rígida, lo que manifiesta una negación al medio físico, al medio natural para el trazado de la nueva ciudad.

Los nuevos conceptos urbanos:

Dentro de las características de las nuevas ciudades del siglo XIX, además de la racionalidad de un planteo acorde y funcional a una realidad económica productora, como hemos visto, es posible detectar en Resistencia algunos elementos propios de la urbanística decimonónica devenidos de modelos higienistas europeos y americanos, que encontraron lugar en la mayor parte de las capitales latinoamericanas y, fundamentalmente, en las nuevas ciudades capitales y administrativas, como pueden ser el caso La Plata en la Argentina; Washington, en los Estados Unidos.

Gran parte de estas nuevas concepciones urbanas se vieron reflejadas en las normas y reglas para la fundación de nuevos poblados, vistas anteriormente, como en las características fisonómicas que fueron adoptando estas ciudades a lo largo de las primeras décadas de formación. Es decir que algunos de estos rasgos se vieron incorporados directamente en la definición de las trazas originales y otros se configuraron acompañando el proceso de consolidación y transformación de pueblos en "ciudades".

\section{La cuadrícula:}

La estructura de la traza de Resistencia, como la de tantas otras ciudades y poblados contemporáneos presenta cierta continuidad con el mo-
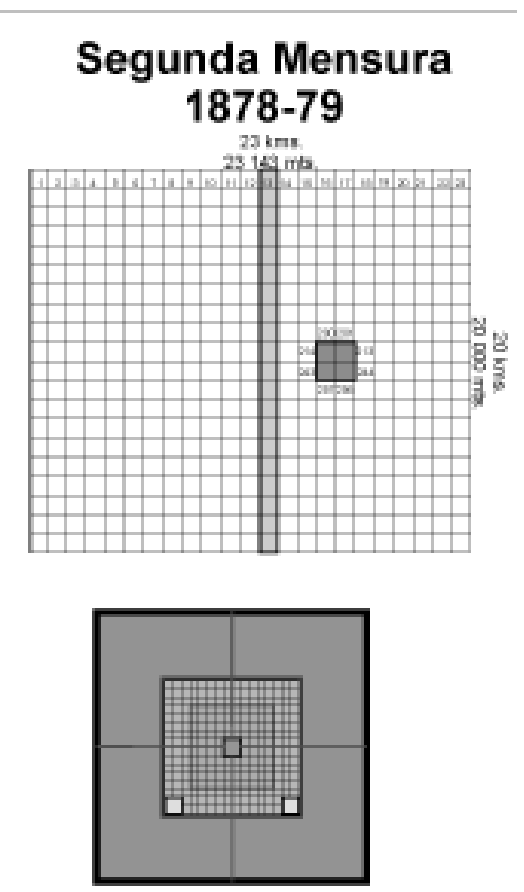

Fig. 4

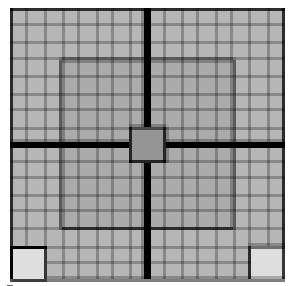




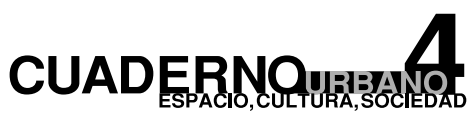

136

\author{
La Colonia Resistencia Como Modelo de Fundación Republicana \\ Procedimientos Fundacionales y Características Urbanas
}
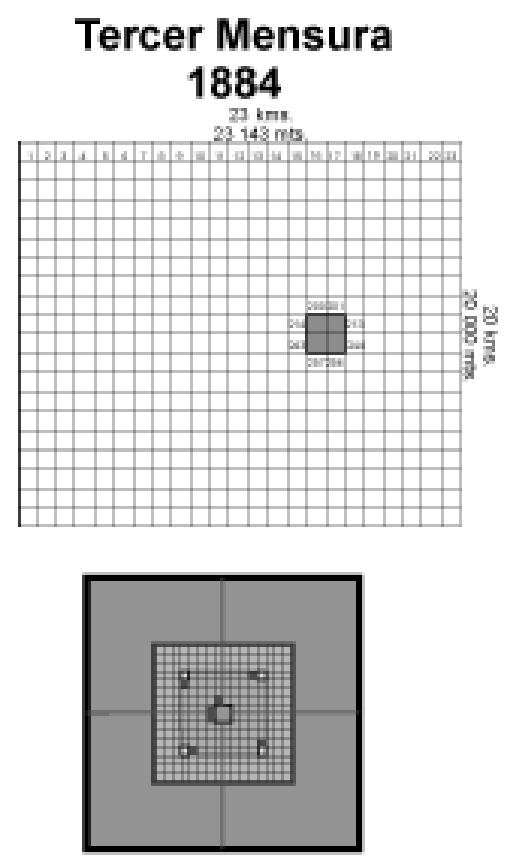

Fig. 5

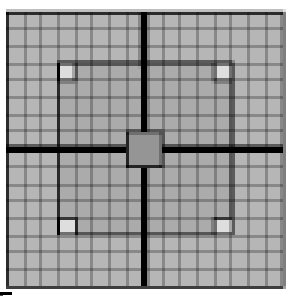

delo hispánico de cuadrícula en lo referente al sector de pueblo. No obstante, la principal diferencia se presenta en la concepción territorial del asentamiento, siendo que no aparece como un centro urbano aislado, rodeado inmediatamente del área rural, sino que aprovecha las virtudes de la cuadrícula para la modulación y la conformación de lo que llamamos la macrocuadrícula territorial a la cual ya nos hemos referido. De esta manera queda por un lado establecida la diferenciación de los usos del suelo en la subdivisión modular de la unidad de chacra (o si se quiere en la progresión modular del los solares urbanos) definiendo las áreas rurales como potenciales extensiones urbanas, por una lado, y por el otro la modulación en cuadrícula otorga continuidad a las áreas productivas en toda la extensión del territorio evitando espacios intersticiales improductivos.

La ciudad de Resistencia quedó definida por una trama totalmente regular, estructurada a partir de ejes ortogonales ubicados a $45^{\underline{0}}$ respecto de los puntos cardinales, y cuyos límites configuraron un cuadrado perfecto de 400 has. La colonia quedaba circunscrita por una estructura en damero, con una plaza central "cuádruple" (Nicolini, 2000: 8) y cuatro en los extremos del perímetro de pueblo; $y$ avenidas principales que se interceptaban en la plaza. (Fig.7)

La disposición de plazas periféricas indica también una intencionalidad de lograr una ciudad policéntrica (recordemos que en las normas para el trazado estaba expuesta esta idea previendo la disponibilidad de lotes ubicados frente a ellas para el desarrollo de actividades públicas).

La jerarquización de vías de circulación:

En la estructura de la ciudad reconocemos una jerarquización de vías de circulación (ya establecidas en las mensuras, calles y avenidas diferen- 


\section{Luciana Sudar Klappenbach}

La Colonia Resistencia Como Modelo de Fundación Republicana

Procedimientos Fundacionales y Características Urbanas

ciadas por el ancho de las mismas) cuyo planteo determina el desarrollo de las vías en dos anillos de circunvalación; a las que se le suman dos ejes que se cruzaban a la manera de cardo y decumano en la plaza central y vinculaban estos anillos con el centro. La estructura circulatoria estaba claramente definida; diferenciaba cada una de las categorías de circulación, rompiendo con la monotonía que presentaba hasta entonces la cuadrícula hispánica (Fig. 8).

En este período se ampliaron las dimensiones dadas a calles y avenidas. Estas (bulevares), adquirieron la siguiente fisonomía: amplias veredas con parterres arbolados, una senda de circulación vial, platabanda intermedia parquizada, segunda senda vial, repitiéndose el esquema en las veredas. La amplitud de las circulaciones vehiculares y peatonales tiene directa correlación con los nuevos usos de los espacios públicos y la incorporación de arbolado urbano y espacios parquizados, concepciones espaciales propias del urbanismo decimonónico, heredadas de la urbanística francesa.

\section{Los espacios verdes: plazas}

Cuatro manzanas centrales se definieron para plaza principal, plaza 25 de Mayo de 1810, y otros cuatro lotes perimetrales para las plazas ubicadas en la intersección del primer anillo de circunvalación. Aparece la plaza como elemento estructurante de las funciones urbanas. En el caso de Resistencia, aunque la ley consignaba la previsión de

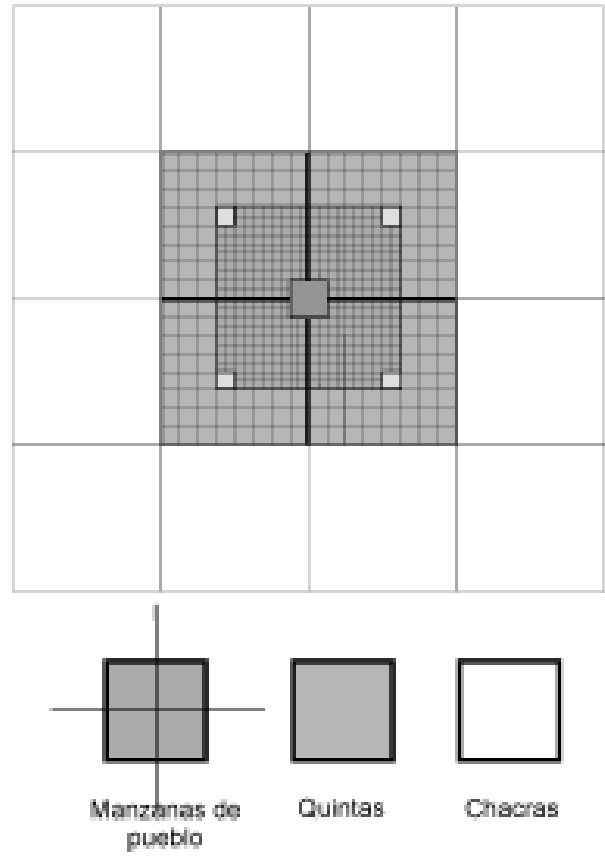

Lotes urbanos y rurales: Marzanas $=100 \mathrm{mts}$. $\mathrm{X} \times 100 \mathrm{mts}$

Solares de pueblo: $50 \mathrm{mts}$. X $50 \mathrm{mts}$ Quintas $100 \mathrm{mts} \times 100 \mathrm{mts}$

Chacras: 1000mts. X X1000mts. 


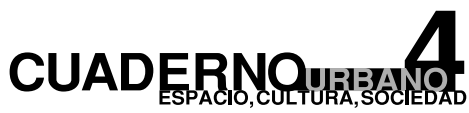

138

\section{La Colonia Resistencia Como Modelo de Fundación Republicana Procedimientos Fundacionales y Características Urbanas}

lotes para edificios públicos adyacentes a cada una de la plazas, la tendencia de centralización convirtió a la plaza central en elemento aglutinante de la funciones urbanas, no siendo así en las perimetrales que esperaron décadas para que por fin se iniciara su urbanización, cuyos espacios se conservaban como lotes vacíos. En estos espacios, que aparecían como ordenadores del sistema urbano, el uso y sentido que se les dio fue diferente al período anterior hispánico: se hace presente el concepto de espacios verdes y de recreación, la idea de pulmón de ciudad. La plaza dejó de ser centro de actividades cívicas y comerciales para transformarse en lugar de paseos y esparcimiento. La plaza 25 de mayo de 1810 tuvo siempre un papel protagónico en la imagen de la ciudad, y en aquellos lejanos años daba vida a la Colonia Resistencia.

\section{Presencia del ferrocarril}

"En esta etapa del siglo, el ferrocarril se transformó en algo más que un medio de transporte: pasó a ser algo así como un símbolo de progreso. También fue uno de los elementos constitutivos de la nueva estructura física de la ciudad argentina". (Nicolini, 2000: 6)

El Ferrocarril Rural fue el primer sistema férreo que incursionó en la ciudad. El Ferrocarril Económico, Ferrocarril rural o Decauville, fue uno de los elementos estructurantes de las actividades y del crecimiento de la ciudad, así como también identificador de una época urbana.

El Ferrocarril Rural, presente en nuestra ciudad desde 1903, fue el medio más efectivo para las comunicaciones interurbanas, por un lado y regional por otro. Con el transcurso de la primera década del siglo se concretaron extensiones de las vías vinculando diversos puntos de la ciudad principalmente relacionados con la actividad económica-productiva: Fábricas, comercios, puerto.

Las vías del Decauville Resistencia - Barranqueras (conocido como "Trencito Dodero" o Ferrocarril Rural) consolidaron el fortalecimiento de este eje a nivel interurbano. Si obser- 


\section{Luciana Sudar Klappenbach}

La Colonia Resistencia Como Modelo de Fundación Republicana

Procedimientos Fundacionales y Características Urbanas

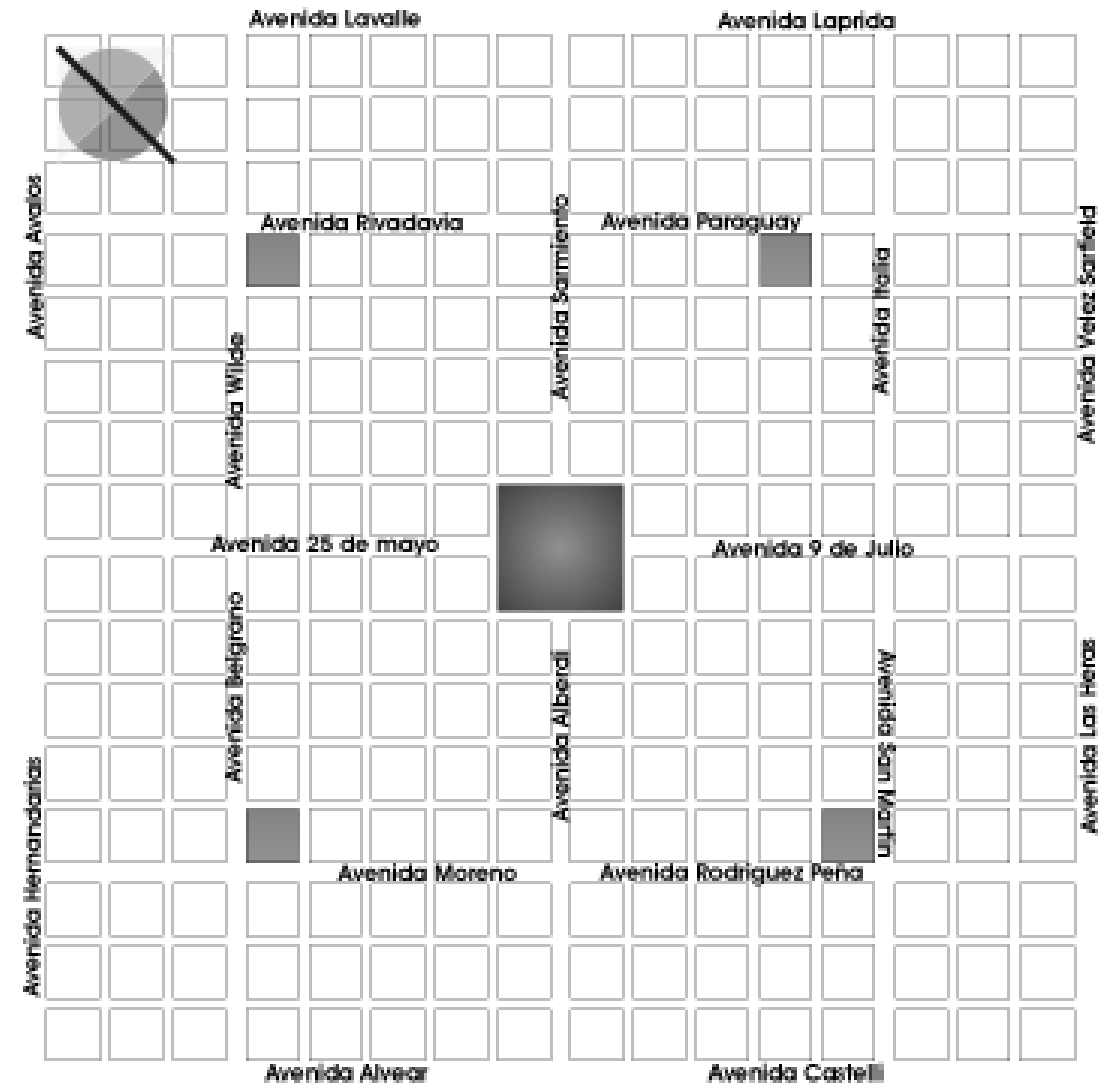

Fig. 7 


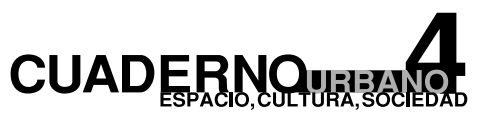

140

\section{La Colonia Resistencia Como Modelo de Fundación Republicana}

Procedimientos Fundacionales y Características Urbanas

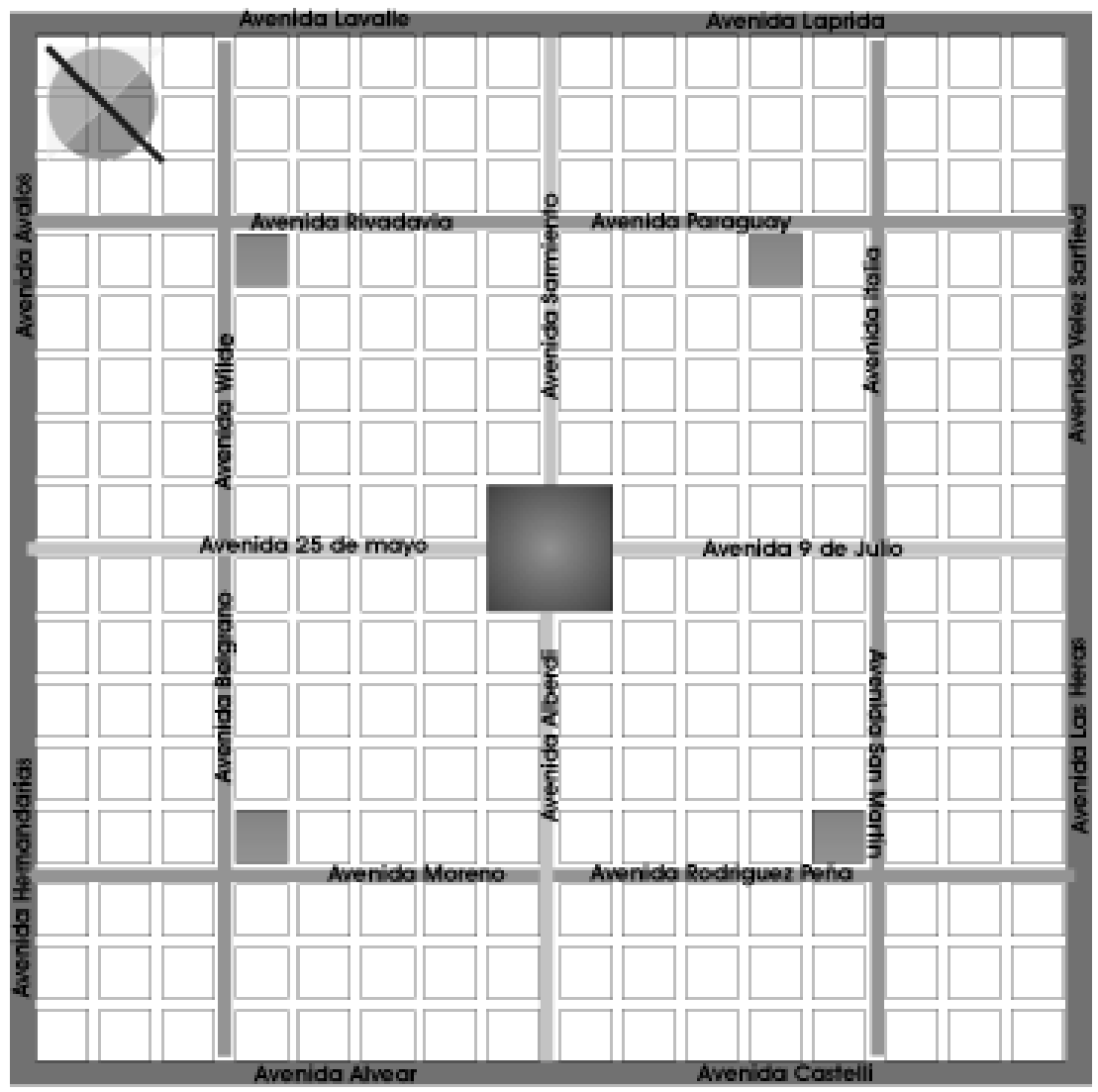

Fig. 8 


\section{Luciana Sudar Klappenbach}

La Colonia Resistencia Como Modelo de Fundación Republicana

Procedimientos Fundacionales y CaRacterísticas Urbanas

vamos la planta urbana con los trazados del ferrocarril rural se evidencia claramente el sentido de los flujos del área, con predominio de un eje longitudinal, y un mayor desarrollo y densificación en el área central, próximo a la Plaza 25 de Mayo.

En Resistencia llegó el primer ferrocarril de conexión interprovincial en 1907, el Ferrocarril Francés de la Provincia de Santa Fe y tendió sus vías en el límite Nordeste de la ciudad, en coincidencia con el trazado de las avenidas Lavalle y Laprida. En 1909 se construyó el Ferrocarril del Estado en límite opuesto al Ferrocarril Francés, también en coincidencia con la arteria sudoeste, avenidas Moreno y Rodríguez Peña. La aparición de los nuevos sistemas de transportes, introducidos en las ciudades argentinas, en las postrimerías del siglo XIX o primeras décadas del siglo XX conformaron ejes y limitantes urbanas, con su inevitable incidencia en el desarrollo de las tramas de la ciudad.

\section{CONCLUSIONES:}

Con este artículo se ha intentado aproximar una idea del proceso de construcción de la ciudad de Resistencia en sus primeros años, considerando para su análisis las particularidades de los trazados del siglo XIX tanto en sus aspectos políticos, económicos, técnicos y disciplinares.

Se ha podido verificar cómo el cambio estructural dentro de la dinámica de la economía internacional, y de los ideales liberales de la generación de gobernantes que llevó adelante el nuevo modelo, definió un sistema de ocupación territorial tanto en lo rural como en lo urbano, aplicando nuevas concepciones en la construcción del territorio. Esta nueva forma de percibir el territorio basada en la aplicación de una macrocuadrícula, sistematizando el accionar sobre cualquier realidad geográfica ha ofrecido además de las ventajas técnicas —que facilitaban las mensuras - ventajas económicas: suponiendo una explotación total del territorio; y políticas: en la posibilidad de una mejor distribución de la tierra, extendiendo la ciudad a toda sección rural cuadriculada (Cacopardo, 2000: 175)9. 


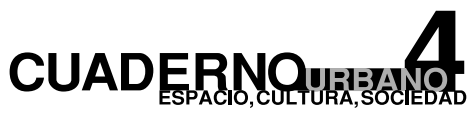

142

\section{La Colonia Resistencia Como Modelo de Fundación Republicana Procedimientos Fundacionales y Características Urbanas}

En Resistencia, la implementación de modelos prototípicos trató de cumplirse lo más estrictamente posible, y si bien existieron condiciones y situaciones locales que no podían acomodarse a lo estipulado por las regulaciones, logró imponerse la "parrilla territorial" sobre la realidad geográfica local, tanto en las áreas destinadas a actividades rurales como en el trazado del pueblo. En este sentido, se pensaba que la mano del hombre podría efectuar los cambios en la naturaleza necesarios para el logro de los fines que éste se proponía, acorde a la cosmovisión del racionalismo científico de los siglos XVIII y XIX. Este pensamiento sin embargo, fue el que ordenó la fundación de las ciudades latinoamericanas ya desde el periodo colonial "crecieron bajo la concepción moderna de relación hombre naturaleza, fueron trazadas a imagen y semejanza unas de otras, sin tener en cuenta las características del medio natural... La actitud ante la naturaleza fue de sometimiento y dominación como forma de expresión de progreso y desarrollo." (Barreto, 1998:26).

Por último, debe destacarse que si bien Resistencia se encuadra, en el orden de la planificación a lo que se llamó "Colonias productivas o agrícolas", debiendo responder más a una cuestión funcional en el orden político y económico con un trazado pensado para servir a estos fines, se puede afirmar que tanto en su concepción como centro urbano, cuanto en su posterior formación y consolidación incorporó las nuevas ideas que proponía el urbanismo del Siglo XIX.

Por todo lo analizado se considera que, más allá de que se tome como caso paradigmático del urbanismo liberal en nuestro país a la ciudad de La Plata (que reunía todas las posibilidades articuladas que ofrecía el urbanismo europeo, especialmente francés), deben incorporarse a esta definición de ciudades liberales o republicanas los asentamientos, como Resistencia, que emergieron en el territorio en este período, que si bien respondían a un "tipo genérico" según el predominio de sus funciones, no permanecieron inertes frente a las nuevas ideas urbanas que se aplicaban a gran parte de las ciudades del mundo en el siglo XIX y principios del XX. 


\section{Luciana Sudar Klappenbach}

La Colonia Resistencia Como Modelo de Fundación Republicana

Procedimientos Fundacionales y CaRacterísticas Urbanas

\section{BIBLIOGRAFÍA:}

Barreto, Miguel Ángel. 1998. "El medio ambiente, el subdesarrollo y la ciudad. Algunas líneas de acción para una política urbana ambientalista y social”. En: Propuesta ecológica. № 21, Posadas.

Cacopardo, F. 2000. "Ciudad y territorio en el siglo XIX: de la macrocuadrícula territorial a la manzana". En: Cuadernos de Historia Urbana I. Tucumán, Facultad de Arquitectura y Urbanismo. Universidad Nacional de Tucumán. ..- 2002. ¿Que hacer con la extensión? Mar del Plata, ciudad y territorio siglos XIX y XX. Madrid - Buenos Aires. Alianza editorial.

Colazo, M. S. 1975. “Resistencia entre 1880 y 1895.” En: Folia Histórica del Nordeste, Nro. 3. Resistencia, Universidad Nacional del Nordeste. Instituto de Historia. Facultad de Humanidades.

Ferrer, A. 1983. La economía argentina. 16 ed. Buenos Aires. Fondo de Cultura Económica.

Gorelik, A. 1998. La Grilla y el Parque. Espacio público y cultura urbana en Buenos Aires. 1887-1936. Buenos Aires. Universidad Nacional de Quilmes.

Halperin Donghi, Tulio.1996. Historia contemporánea de América Latina. 14 Ed. Madrid, Alianza Editorial.

Leiva, M. E.1981. "Las Colonias del área chaqueña”. En:2C Construcción de la Ciudad. Barcelona, Romargraf S. A.

Maeder, E. 1996. Historia el Chaco. Buenos Aires, Editorial Plus Ultra,

Miranda, G 1968. "Etapas fundacionales de Resistencia." En: “Aportes para una historia del Chaco". , Resistencia, Biblioteca El Territorio.

Nicolini, A. 2000. "Las cuatro etapas de la ciudad Argentina, según su estructura, funciones y paisajes urbanos". Sexto Congreso Nacional y Regional de Historia Argentina. Buenos Aires, Academia Nacional de la Historia.

Piacentini, C. P. 1970. Historia de la Provincia del Chaco. Buenos Aires, Chiman, SA

Rossi, E.1997. "Identidad del Chaco." Resistencia, Meana y Meana.

Schaller, E. C.1986. La colonización en el territorio Nacional del Chaco en el período 1869 - 1921. Resistencia. IGHI, UNNE. Shumway, N. 2002. La invención de la Argentina. Buenos Aires, Emecé Editores S.A.

Vázquez Gualtieri, J. 1978. "Resistencia, a 32 Años del 2000." Aportes para la Historia del Chaco. Biblioteca "El Territorio" 1: 83-90. Resistencia. 